\title{
Possible Role of ZPAC, Zygote-specific Proteasome Assembly Chaperone, During Spermatogenesis in the Mouse
}

\author{
Natsumi SHIMIZU1), Kimihiro UENO2), Ena KURITA2), Seung-Wook SHIN²), \\ Takuji NISHIHARA ${ }^{1)}$, Tomoko AMANO ${ }^{1}$, , Masayuki ANZAI ${ }^{1,4)}$, Satoshi KISHIGAMI ${ }^{1}$, \\ Hiromi KATO ${ }^{1,4)}$, Tasuku MITANI ${ }^{1,4)}$, Yoshihiko HOSOI'1) and Kazuya MATSUMOTO ${ }^{1)}$ \\ 1) Laboratory of Molecular Developmental Biology, Graduate School of Biology-Oriented Science and Technology, Kinki \\ University, Wakayama 649-6493, Japan \\ ${ }^{2}$ Laboratory of Molecular Developmental Biology, Faculty of Biology-Oriented Science and Technology, Kinki University, \\ Wakayama 649-6493, Japan \\ 3) Laboratory of Cellular and Developmental Biology, NIDDK, National Institutes of Health, Maryland 20892, USA \\ 4) Institute of Advanced Technology, Kinki University, Wakayama 642-0017, Japan
}

\begin{abstract}
In the mammalian testis, the ubiquitin-proteasome system plays important roles in the process that promotes the formation of mature sperm. We recently identified zygote-specific proteasome assembly chaperone (ZPAC), which is specifically expressed in the mouse gonads and zygote. ZPAC mediates a unique proteasome assembly pathway in the zygote, but the expression profile and function of ZPAC in the testis is not fully understood. In this study, we investigated the possible role of ZPAC during mouse spermatogenesis. First, we analyzed the expression of ZPAC and 20S proteasome subunit $\alpha 4 /$ PSMA7 in the adult mouse testis. ZPAC and $\alpha 4$ were expressed in spermatogonia, spermatocytes, and round spermatids. In elongating spermatids, ZPAC was expressed until step 10, whereas expression of $\alpha 4$ persisted until step 12 . We then examined the expression profile of ZPAC and $\alpha 4$ in a mouse model of experimental unilateral cryptorchidism. Consistent with appearance of morphologically impaired germ cells following cryptorchidism, the ZPAC protein level was significantly decreased at 4 days post induction of experimental cryptorchidism (D4) compared with the intact testis, although the amount of $\alpha 4$ protein persisted at least until D10. Moreover, intense ZPAC staining was co-localized with staining of annexin V, an early indicator of apoptosis in mammalian cells, in germ cells of cryptorchid testis, but ZPAC was also expressed in germ cells showing no detectable expression of annexin V. These results suggest that ZPAC plays a role during spermatogenesis and raises the possibility that $20 \mathrm{~S}$ proteasome mediated by ZPAC may be involved in the regulation of germ cell survival during spermatogenesis.
\end{abstract}

Key words: Spermatogenesis, Ubiquitin-proteasome system, Zygote-specific proteasome assembly chaperone (ZPAC)

(J. Reprod. Dev. 60: 179-186, 2014)

$\mathbf{T}$ he $26 \mathrm{~S}$ proteasome is the main protease for the proteolytic elimination of regulatory proteins or damaged proteins in eukaryotic cells. For proteolytic elimination, a substrate protein is generally mediated by ubiquitination, the covalent attachment of the small polypeptide ubiquitin to lysine residues within a target protein. The ubiquitin-proteasome system (UPS) plays a central role in cellular homeostasis, DNA repair, apoptosis, immune response, signal transduction, transcription, metabolism, protein quality control and development programs [1-3].

The $26 \mathrm{~S}$ proteasome is a highly conserved protein degradation machine made up of two complexes: the catalytic 20S proteasome, also called the core particle (CP), and the 19S regulatory particle (RP), both of which are assembled from a set of multiple distinct subunits [1]. Interestingly, this diversification was considered to be not necessary in light of the general housekeeping feature of the

Received: January 7, 2014

Accepted: January 20, 2014

Published online in J-STAGE: February 28, 2014

(C)2014 by the Society for Reproduction and Development

Correspondence: K Mastumoto (e-mail: kazum@waka.kindai.ac.jp)

UPS in the degradation of regulatory proteins or damaged proteins. However, existence of a tissue-specific assembly strategy with tissue-specific subunits or regulators has been demonstrated [4-6]. Recently, we identified a molecule, named ZPAC for zygote-specific proteasome assembly chaperone, that is specifically expressed in the mouse ovary and testis, and the mouse expression of ZPAC as well as the proteasome activity is transiently increased at the maternal-to-zygotic transition [7, 8]. ZPAC directly forms a complex with ubiquitin-mediated proteolysis $\underline{1}$ (Ump1), which is an assembly chaperone that facilitates the formation of $20 \mathrm{~S}$ proteasome, and is specifically associated with precursor forms of the $\beta$ subunits of CP. Knockdown of ZPAC in early embryos causes a significant reduction in $\mathrm{CP}$ formation and causes accumulation of precursors of the $\beta$ subunits, resulting in arrest of embryonic development. However, further details about the expression profile of ZPAC and function of $20 \mathrm{~S}$ proteasome mediated by ZPAC in the mouse testis still remain undetermined.

In the testis, the differentiation process from spermatogonia to mature sperm is a highly integrated testicular homeostasis that includes mitosis, meiosis and morphological changes, in which the UPS plays a key role [9]. In fact, aberrant function of UPS-associated genes has 
resulted in defective spermatogenesis, which impairs male fertility [10-12]. Furthermore, a previous report indicated that loss of the proteasome activator PA200, which is abundantly expressed in the testis, results in a severe reduction in male fertility due to failure of germ cells to progress through meiosis, although the molecular mechanism remains largely unknown [12]. Recent work shows that most testis-specific proteasomes, which contain PA200 and are called as spermatoproteasomes, induce the degradation of histones in the histone-to-protamine exchange during spermatogenesis [13].

Cryptorchidism results in arrest of spermatogenesis due to the loss of germ cells from the testis, and apoptosis has been shown to be the primary mechanism of germ cell loss in cryptorchidism [14]. Generally, the UPS plays an important role in the regulation of apoptosis by targeting key cell death proteins [15]. In fact, based on the function of polyubiquitination or deubiquitination in cryptorchid mouse testes, the UPS is suggested to be involved in the regulation of germ cell survival during spermatogenesis [16-18]. However, the possible role of ZPAC in apoptosis during spermatogenesis still remains uncertain. Thus, to investigate the possible role of ZPAC during spermatogenesis in the mouse, we examined the expression profile of ZPAC in normal and experimental cryptorchid testes. Because the $\mathrm{CP}$ is composed of 28 subunits arranged in a cylindrical particle as four heteroheptameric rings, $\alpha_{1-7} \beta_{1-7} \beta_{1-7} \alpha_{1-7}[1]$, we also compared the expression profile between ZPAC and 20S proteasome subunit $\alpha 4 /$ PSMA7 proteins in these testes.

\section{Materials and Methods}

\section{Animals}

All mice (ICR strain) were purchased from Kiwa Experimental Animals (Wakayama, Japan) at over 8 weeks of age and maintained in light-controlled, air-conditioned rooms. This study was carried out in strict accordance with the recommendations in the Guidelines of Kinki University for the Care and Use of Laboratory Animals. The protocol was approved by the Committee on the Ethics of Animal Experiments of Kinki University (Permit Number: KABT-19-003). All mice were killed by cervical dislocation, and all efforts were made to minimize suffering and to reduce the number of animals used in the present study.

\section{Experimental unilateral cryptorchidism}

Experimental cryptorchidism was induced as described previously [19]. In brief, adult male mice were anesthetized with sodium pentobarbital (Somnopentyl, Kyoritsu, Tokyo, Japan), and a small longitudinal incision was made on the abdomen. The left testis was manipulated into the abdomen and sutured to the abdominal wall. The abdominal and scrotal testes were collected at 1, 4, 7 and 10 days postoperatively. The scrotal right testis was used as a control (intact testis). The testes were weighted before the following experiments.

\section{Immunohistochemical analysis}

The procedures were essentially performed using a commercial kit (Dako, Copenhagen, Denmark; EnVision System-HRP kit, K1390) as prescribed by the manufacturer. In brief, sections of testes fixed with $4 \%$ paraformaldehyde were deparaffinized, dehydrated and incubated with methanol containing $0.3 \% \mathrm{H}_{2} \mathrm{O}_{2}$ at room temperature
(RT) for 10 min. After being washed with phosphate-buffer saline (PBS), the sections were incubated in $50 \mathrm{mM}$ Tris- $\mathrm{HCl}(\mathrm{pH} 7.4)$ containing $1 \%$ bovine serum albumin (BSA) for $30 \mathrm{~min}$ at RT. Then, the sections were incubated with anti-ZPAC polyclonal antibody (final dilution, 1:100) or anti- $\alpha 4$ subunit monoclonal antibody (final dilution, 1:5,000; Enzo Life Science, Farm ingdale, NY, USA; BMLPW9140) in a solution composed of $50 \mathrm{mM}$ Tris- $\mathrm{HCl}(\mathrm{pH} 7.4)$ and $1 \% \mathrm{BSA}$ at RT for $1 \mathrm{~h}$. After three washes with PBS, the sections were incubated with peroxidase labelled polymer conjugated to goat anti-rabbit and goat anti-mouse immunoglobulins in Tris-HCL buffer (Dako; K1390) or polyclonal goat anti-rabbit immunoglobulins (final dilution, 1:500; Dako; P0448) for anti-ZPAC and polyclonal goat anti-mouse immunoglobulins (final dilution, 1:500; Dako; P0447) for anti- $\alpha 4$ subunit for $1 \mathrm{~h}$ at RT, respectively. After three washes with PBS, the sections were incubated with substrate-chromogen solution for $5 \mathrm{~min}$ at RT. Finally, the slides were imaged using an Olympus BX51 microscope (Olympus, Tokyo, Japan) equipped with an Olympus DP70 digital camera (Olympus). At least three independent experiments were performed for each group.

\section{Histological analysis}

The specimens were fixed for $48 \mathrm{~h}$ in $4 \%$ paraformaldehyde solution and then dehydrated by ethanol and embedded in paraffin. The $5 \mu$ m-thick sections were routinely deparaffinized, dehydrated and treated with hematoxylin and eosin. Subsequently, the slides were imaged using an Olympus BX51 microscope (Olympus) equipped with an Olympus DP70 digital camera (Olympus). At least three independent experiments were performed for each group.

\section{Proteasome activation assay}

Peptidase activity of the specimens was measured by using a fluorescent peptide substrate, succinyl-Leu-Leu-Val-Tyr-7-amino4-methylcoumarin (Suc-LLVY-AMC), as described previously [20]. At least two or three independent experiments were performed for each group.

\section{Western blot analysis}

The procedures were essentially performed as described previously [7, 21-24]. In brief, $25 \mu \mathrm{g}$ of protein extracts from whole testis were subjected to sodium dodecyl sulfate (SDS) polyacrylamide gel electrophoresis. The protein extracts were resolved in 10\% running gels and electrophoretically transferred to polyvinylidene difluoride (PVDF) membranes (GE Healthcare, Little Chalfont, UK). The membranes were incubated in Block Ace (Dainippon Sumitomo Pharma, Osaka, Japan) at RT for $1 \mathrm{~h}$. They were washed with PBS containing $0.2 \%$ Tween 20 (PBST) and incubated at $4 \mathrm{C}$ overnight with anti-ZPAC antibody (final dilution, 1:5,000), anti- $\alpha 4$ subunit antibody (final dilution, 1:10,000) or with anti-actin antibody (final dilution, 1:50,000; Santa Cruz Biotechnology, Heidelberg, Germany; sc-1616) as a loading control. The membranes were washed in PBST, incubated with donkey anti-rabbit IgG horseradish peroxidase (HRP) conjugate (1:50,000; Millipore, Billerica, MA, USA; AP182P) for anti-ZPAC, donkey anti-mouse IgG HRP conjugate $(1: 50,000$; Millipore; AP192) for anti- $\alpha 4$ subunit or donkey anti-goat IgG HRP conjugate (1:50,000; Millipore Corp.; AP180P) for anti-actin at RT for $1 \mathrm{~h}$, washed with PBST and developed using ECL Prime Western 
Blotting detection reagent (GE Healthcare).

\section{Immunofluorescence analysis}

Sections of specimens fixed with $4 \%$ paraformaldehyde were deparaffinized and dehydrated. After antigen activation with $10 \mathrm{mM}$ citrate buffer ( $\mathrm{pH} 7.0$ ), the sections were incubated in $50 \mathrm{mM}$ Tris- $\mathrm{HCl}$ ( $\mathrm{pH}$ 7.4) containing $1 \%$ BSA at RT for $1 \mathrm{~h}$. Then, the sections were incubated with both anti-ZPAC polyclonal antibody (final dilution, 1:100) and anti-annexin V (R-20) polyclonal antibody (final dilution, 1:500; Santa Cruz Biotechnology; sc-1929) in a solution composed of $50 \mathrm{mM}$ Tris-HCL and 1\% BSA overnight at $4 \mathrm{C}$. After three washes with PBS, the sections were incubated with Alexa Fluor 594-labeled donkey anti-rabbit IgG (final dilution, 1:2,000; Invitrogen, Carlsbad, CA, USA; A-21207) for anti-ZPAC and Alexa Fluor 488-labeled donkey anti-goat $\mathrm{IgG}$ antibody (final dilution, 1:2,000; Invitrogen; A-11055) for anti-annexin $\mathrm{V}$ in $50 \mathrm{mM}$ Tris- $\mathrm{HCl}(\mathrm{pH}$ 7.4) containing $1 \% \mathrm{BSA}$ at RT for $1 \mathrm{~h}$. Subsequently, the sections were washed three times with PBS and mounted in Vectashield mounting medium (Vector Laboratories, Burlingame, CA, USA) containing 2-5 $\mu \mathrm{g}$ / ml DAPI (Invitrogen; D1306). Finally, fluorescence images were obtained using an Olympus BX51 microscope (Olympus) equipped with an Olympus DP70 digital camera (Olympus). At least three independent experiments were performed for each group.

\section{Statistical analysis}

For statistical analysis, we used StatView version 5.0 (SAS Institute, Cary, NC, USA) and Microsoft Excel and performed analysis of variance (ANOVA) with an $\alpha$ level of 0.05 to determine possible statistically significant differences between group means.

\section{Results}

\section{Protein expression profile of ZPAC and 20S proteasome} subunit $\alpha 4 / P S M A 7$ in normal mouse testes

Our previous study showed that ZPAC protein was detected at least in mouse spermatogonia [7]. To further elucidate the cell-type- and developmental stage-specific localization of ZPAC and $\alpha 4$ proteins in the adult mouse testis in more detail, we examined the expression of ZPAC and $\alpha 4$ proteins in germ cells at various stages of seminiferous tubules classified according to the criteria as described by Russell et al. [25]. In this line of analysis, we first observed the reactivity of anti-ZPAC in Leydig and Sertoli cells, but these signals were considered to be nonspecific because the in situ hybridization analysis in our previous study showed no signals of ZPAC mRNA in Leydig and Sertoli cells in mice [7]. In addition, faint $\alpha 4$ immunoreactivity was observed in Leydig and Sertoli cells. As shown in Fig. 1A and $\mathrm{B}, \mathrm{ZPAC}$ and $\alpha 4$ proteins were expressed in both the cytoplasm and nucleus of germ cells. In spermatogonia, ZPAC protein is moderately expressed, similar to $\alpha 4$ protein. The expressions of ZPAC and $\alpha 4$ proteins were observed in characteristic patterns in spermatocytes, round spermatids and elongating spermatids (Fig. 1C). Relatively intense signals for both ZPAC and $\alpha 4$ proteins were also detected from spermatocytes up to step 10 elongating spermatids. Thereafter, ZPAC expression dispersed in step 11 elongating spermatids, whereas the expression of $\alpha 4$ protein persisted up to step 12 elongating spermatids. Taken together, these results suggest that ZPAC plays
A

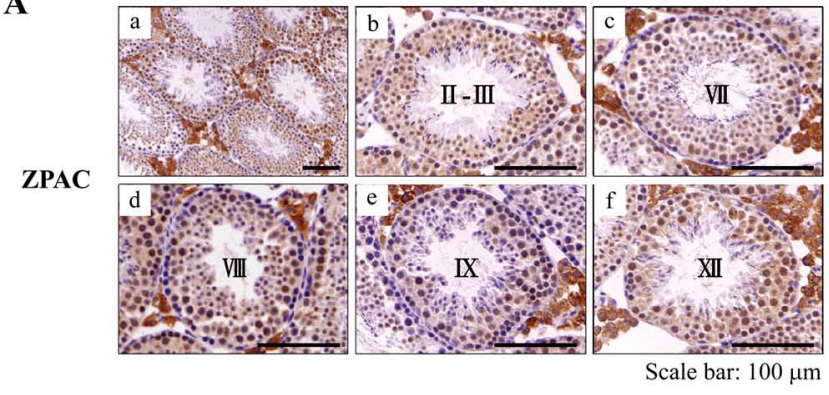

B

$\alpha 4$

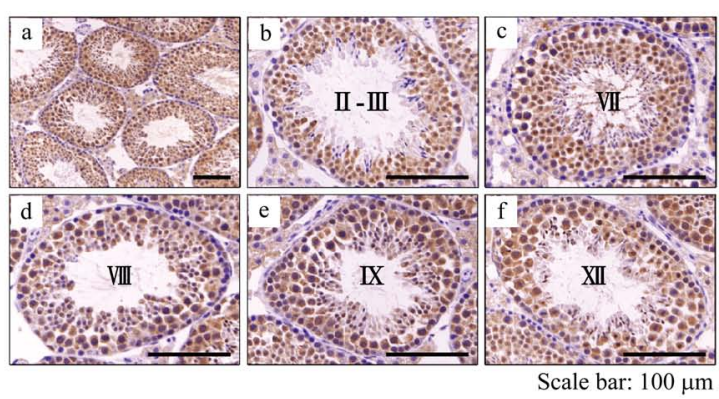

C

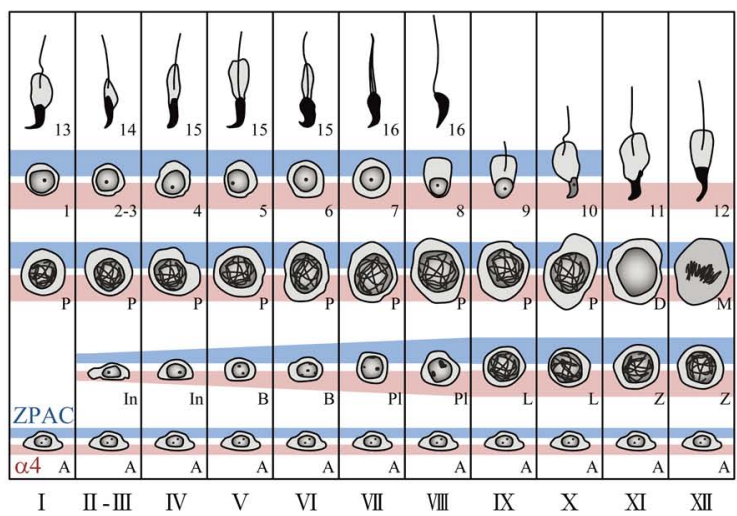

Fig. 1. Immunohistochemical analysis of ZPAC and $\alpha 4$ proteins in the mouse testis. (A) Cell-type- and developmental stage-specific localization of ZPAC protein in the mouse testis. ZPAC-positive cells in the seminiferous tubules are indicated by brown color. Representative seminiferous tubular cross sections showing ZPAC expression at low (a) and high magnification (b-f): II-III stage (b), VII stage (c), VIII stage (d), IX stage (e) and XII stage (f) of spermatogenesis. Nonspecific staining of interstitial/Leydig cells is visible between seminiferous tubules (see in results section). Scale bars represent $100 \mu \mathrm{m}$. (B) Cell-type- and developmental stage-specific localization of $\alpha 4$ in the mouse testis. $\alpha 4$-positive cells in the seminiferous tubules are indicated by brown color. Representative seminiferous tubular cross sections showing $\alpha 4$ expression at low (a) and high magnification (b-f): II-III stage (b), VII stage (c), VIII stage (d), IX stage (e) and XII stage (f) of spermatogenesis. Scale bars represent $100 \mu \mathrm{m}$. (C) Diagram of developmental stage-specific expression of ZPAC (blue) and $\alpha 4$ (pink) during the cycle of the seminiferous epithelium of the mouse. Spermatogonia consist of type A (A), intermediate-type (In) and type B (B) spermatogonia that divide during stages I-XII. Spermatocytes at the $\mathrm{G} 1$ and $\mathrm{S}$ phases are indicated as preleptotene $(\mathrm{Pl})$, and then traverse the meiotic prophase via the leptotene $(\mathrm{L})$, zygotene (Z), pachytene (P) and diplotene (D) phases. Following the meiotic divisions $(\mathrm{M})$, formed spermatids develop in 16 steps into spermatozoa that are shed into the lumen of the seminiferous tubules. 
a role in regulating germ cell development during spermatogenesis and that ZPAC may not be involved in assembly of $20 \mathrm{~S}$ proteasome in step 11-12 elongating spermatids.

\section{Protein expression profile of ZPAC and $20 S$ proteasome} subunit $\alpha 4 / P S M A 7$ in intact and cryptorchid mouse testes

In experimental cryptorchidism, it is suggested that superfluous ROS induced by heat stress can cause oxidative damage in proteins and that the UPS facilitates the degradation of damaged proteins [16]. Thus, to investigate the effect of cryptorchidism on the expression of ZPAC and $\alpha 4$ proteins in the adult mouse testis, we analyzed the expression of ZPAC and $\alpha 4$ proteins in germ cells at various stages of seminiferous tubules in intact and experimental cryptorchid mouse testes (Fig. 2A and Fig. 2B).

First, we examined whether the induction of experimental cryptorchidism resulted in impaired spermatogenesis. During the experimental period, while the weight of the intact testis was unchanged, the weight of the cryptorchid testis was also unchanged at 1 day post induction of experimental cryptorchidism (D1) and then significantly decreased at D4, D7 and D10, resulting in an overall weight loss of more than $60 \%$ in the cryptorchid testis (Fig. 2C). To characterize the morphology of seminiferous tubules of testes subjected to heat stress after cryptorchidism, histological analysis was performed (Fig. 2D). As shown in previous reports [14, 26], the characteristics of spermatogenesis altered by heat stress (for example, nuclear pyknosis, cellular shrinkage, multinucleated giant cells, shrinkage of seminiferous tubule diameter, thin seminiferous epithelium and vacuoles) was observed in experimental cryptorchidism at D4, D7 and D10 compared with in the intact testis. In particular, numerous of multinucleated giant cells were observed.

Next, we analyzed the proteasomal chymotrypsin-like activity from crude lysate of intact and experimental cryptorchid testes on D1, D4, D7 and D10 (Fig. 3A). The evident effect of cryptorchidism on the proteasomal chymotrypsin-like activity in the mouse testis was eventually observed at D10 (experimental cryptorchidism vs. intact testis, $0.86 \pm 0.01$ vs. $1.00 \pm 0.00, \mathrm{P}<0.05$; Ave. $\pm \mathrm{SE}$ ). Furthermore, we examined the expression of ZPAC and $\alpha 4$ proteins in intact and experimental cryptorchid testes on D1, D4, D7, and D10 (Fig. 3B). Consistent with the observation of apparent morphological changes following experimental cryptorchidism (Fig. 2C and 2D), no difference was observed in ZPAC protein level between intact and cryptorchid testes at D1, and thereafter, ZPAC protein levels in the cryptorchid testes at D4, D7 and D10 were significantly decreased by $79 \%$ (experimental cryptorchidism vs. intact testis, $0.79 \pm 0.07$ vs. $1.00 \pm 0.00, \mathrm{P}<0.05$; Ave. $\pm \mathrm{SE}$ ), $29 \%$ (experimental cryptorchidism vs. intact testis, $0.29 \pm 0.05$ vs. $1.00 \pm 0.00, \mathrm{P}<0.05$; Ave. \pm SE) and $65 \%$ (experimental cryptorchidism vs. intact testis, $0.65 \pm 0.01$ vs. $1.00 \pm 0.00, \mathrm{P}<0.05$; Ave. $\pm \mathrm{SE}$ ) compared with intact testes on each day, respectively (Fig. 3C, left). Interestingly, there was no significant difference in $\alpha 4$ protein levels between intact and cryptorchid testes at D1, D4, D7 and D10 (Fig. 3C, right). Therefore, since the proteasome chymotrypsin-like activity reflects the relative activity of UPS, these results raise the possibility that ZPAC may be involved in the regulation of germ cell survival during spermatogenesis and that UPS may also associated with impaired spermatogenesis.
Next, we examined the cell-type- and developmental stage-specific localization of ZPAC and $\alpha 4$ proteins in experimental cryptorchidism. Consistent with the immunoblot analysis, intense signals of ZPAC and $\alpha 4$ proteins were detected in germ cells at D1. However, as the experiment progressed, ZPAC signals were decreased at D4 and were barely detectable at D7, whereas $\alpha 4$ signals were sustained at D4 and D7. This result suggests that ZPAC and $\alpha 4$ have a somewhat different role in the mouse testis following experimental cryptorchidism (Fig. 4A). Indeed, although both ZPAC and $\alpha 4$ protein signals were detected in spermatocytes present in the inner region of seminiferous tubules at D4, $\alpha 4$ protein signals, but not ZPAC protein signals, were observed in multinucleated giant cells, which are known to be undergoing apoptosis [14] at D7 (Fig. 4A).

Apoptosis has been considered the major molecular mechanism of germ cell loss in experimental cryptorchidism [14, 27]. We observed in our experimental cryptorchidism model that ZPAC expression was moderately increased at D1 (Fig. 3C). Thus, to elucidate whether ZPAC might exert its effect in the early stage of testicular germ cell apoptosis induced by experimental cryptorchidism, we examined the co-localization of ZPAC and annexin V, an early indicator of apoptosis in mammalian cells, in the intact and cryptorchid testis. As a result, we found that intense signals of ZPAC proteins were detected in annexin V-positive germ cells of the cryptorchid testis compared with the intact testis, but ZPAC was also expressed in a large number of germ cells showing no detectable expression of annexin $\mathrm{V}$ in the intact and cryptorchid testes (Fig. 4B). This result indicates that ZPAC may be associated with regulation of germ cell survival during ongoing impaired spermatogenesis in experimental cryptorchidism.

\section{Discussion}

In this study, we showed that ZPAC, zygote-specific 20S proteasome assembly chaperone, is expressed in germ cells during spermatogenesis except in elongating spermatids at steps 11-12 and elongated spermatids and that ZPAC may be responsible for regulating germ cell survival during spermatogenesis.

Mammalian spermatogenesis is a highly controlled process in which a precise homeostasis for germ cell proliferation, differentiation and death is maintained. During spermatogenesis, apoptosis regulates germ cells numbers and eliminates aberrant germ cells under a variety of specific conditions [28]. For instance, more than $75 \%$ of germ cells during spermatogenesis die by apoptosis [29]. In particular, apoptosis in spermatocytes and round spermatids is thought to remove cells having errors during meiosis and unfit cells, respectively [30]. The UPS, in general, plays an essential role in the regulation of apoptosis by targeting anti- or pro-apoptotic proteins [15]. There is also evidence that the UPS is involved in the control of germ cell loss during spermatogenesis [16-18]. Our data may provide evidence for the possible role of $20 \mathrm{~S}$ proteasome biogenesis mediated by ZPAC in germ cell survival during spermatogenesis.

Furthermore, it is possible that a tissue-specific $20 \mathrm{~S}$ proteasome mediated by ZPAC is not only involved in germ cell survival but may also be applicable to the process of germ cell apoptosis induced by experimental cryptorchidism. To date, the mechanism of cryptorchidism-induced degenerative changes of the seminiferous 
A



B

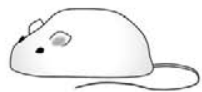

Mice induced unilateral cryptorchidism

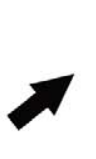

Preparation of histological sections of testes

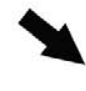

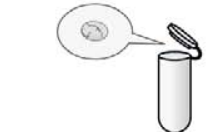

Protein extraction of testes

\section{Examined by}

Histological analysis Immunohistochemial analysis - ZPAC

- $\alpha 4$

Immunofluorescence analysis

- ZPAC

- Annexin V

Examined by

Proteasome activation assay Immunoblot analysis

- ZPAC

- $\alpha 4$
C

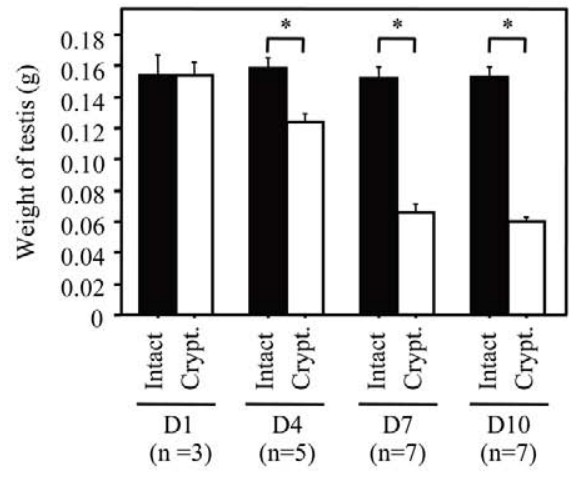

D

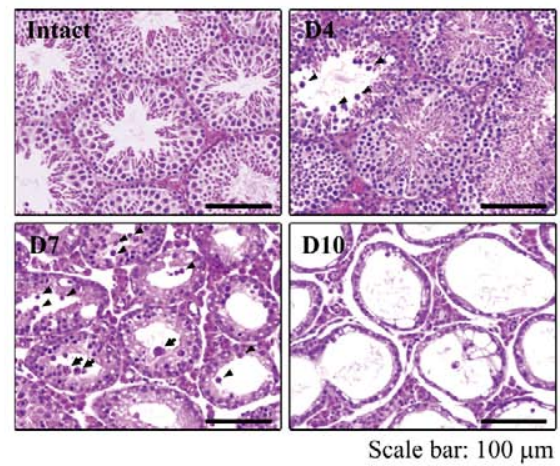

Fig. 2. Schematic diagram of experiments using a mouse model of experimental unilateral cryptorchidism. (A) Schematic diagram of the experimental schedule. (B) Scheme of the experimental procedures. (C) Change in weight of intact and experimental cryptorchid testes. Bars represent the standard error of the mean. The asterisk indicates statistically different values $(\mathrm{P}<0.05)$. m: Intact. $\square$ : Experimental cryptorchidism (Crypt.). (D) Histological sections of intact and experimental cryptorchid testes stained with hematoxylin and eosin. Scale bars represent $100 \mu \mathrm{m}$. Arrowheads and arrows indicate typical cells with nuclear pyknosis/cellular shrinkage and multinucleated giant cells, respectively.

epithelium and elimination of abnormal germ cells are still not fully understood, although the cellular and physiological responses to heat stress have been well demonstrated [31,32]. Interestingly, biochemical analysis of oxidative stress and DNA array data analysis indicate that the dynamics of changes in reactive oxygen species (ROS) production caused by experimental cryptorchidism, in which more effective antioxidative capability was sustained sustained during at least the first 4 days postoperatively and suddenly decreased at day 5 postoperatively, were verified by gene expression data [33]. Since the proteasome plays an important role in selectively degrading oxidatively modified proteins, either in ubiquitin-dependent or ubiquitin-independent manners [34], we speculate that cell-type- or tissue-type-specific $20 \mathrm{~S}$ proteasome mediated by ZPAC might play a role in the early stage of experimental cryptorchidism. Indeed, our previous study demonstrates that ZPAC gene expression is directed by transcription activation in a cellular response to the increased demands of proteasomal degradation of proteins (for example, oxidative stress) [7]. Further studies are needed to confirm our hypothesis.

In conclusion, understanding the function of the UPS including ZPAC during spermatogenesis will help elucidate the molecular mechanisms governing the development of spermatogonia into mature sperm. 
A

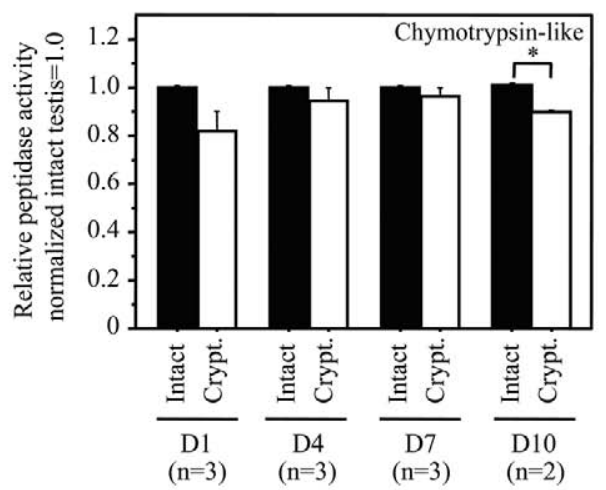

B

\begin{tabular}{|c|c|c|c|c|c|c|c|}
\hline & \multicolumn{2}{|c|}{ D1 } & \multicolumn{2}{|c|}{ D4 } & \multicolumn{2}{|c|}{ D7 } & D10 \\
\hline & Intact & Crypt. & Intact & Crypt. & Intact & Crypt. & Intact Crypt. \\
\hline & $\# 1 \quad \# 2 \quad \# 3$ & $\# 1 \quad \# 2 \quad \# 3$ & $\# 1 \quad \# 2 \# 3$ & $\# 1 \# 2 \quad \# 3$ & $\# 1 \quad \# 2 \quad \# 3$ & $\# 1 \quad \# 2 \quad \# 3$ & $\# 1 \quad \# 2 \quad \# 1 \quad \# 2$ \\
\hline & $-\infty-\infty$ & $-5=$ & $-\infty=2$ & $8=0$ & 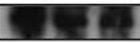 & 5. & 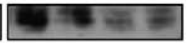 \\
\hline & -00 & 000 & $-\infty$ & 00 & 20 & $3-0$ & $-6-0$ \\
\hline & $-\infty$ & - & $-\infty$ & - & & $\infty$ & $=$ \\
\hline
\end{tabular}

C
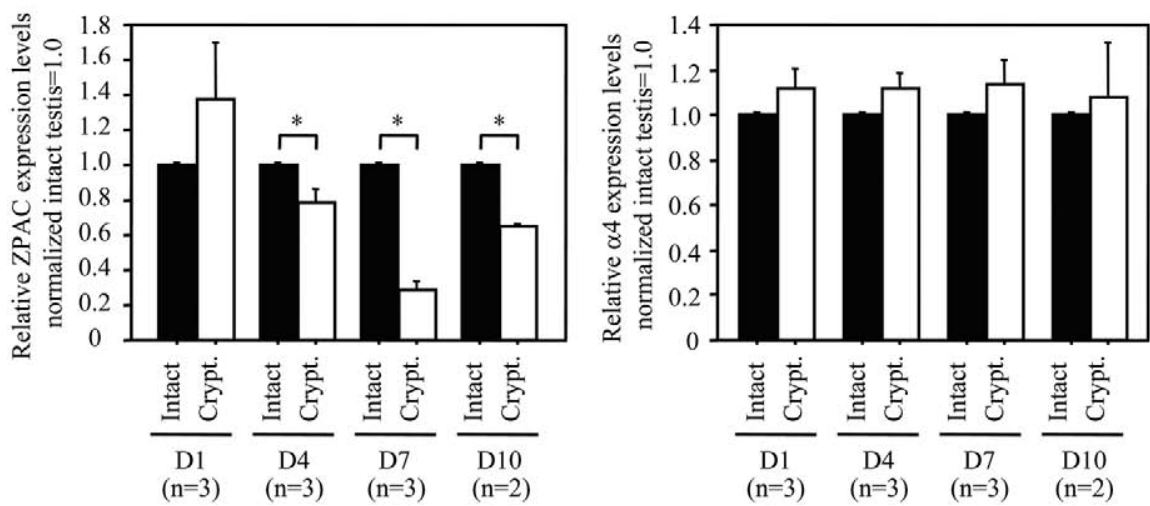

Fig. 3. Proteasome activity analysis and immunoblot analysis of ZPAC and $\alpha 4$ proteins in the cryptorchid mouse testis. (A) Effect of cryptorchidism on the proteasomal chymotrypsin-like activity in the mouse testis. Bars represent the standard error of the mean. The asterisk indicates statistically

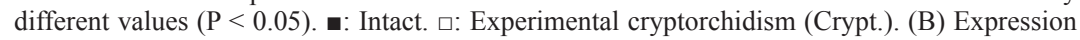
of ZPAC and $\alpha 4$ proteins in intact and experimental cryptorchid testes. $\beta$-actin was used as a loading control. (C) Effect of cryptorchidism on the expression of ZPAC and $\alpha 4$ proteins in the mouse testis. Quantification of the ratios (experimental cryptorchidism/intact) of ZPAC and $\alpha 4$ intensity in Fig. 3B. Bars represent the standard error of the mean. The asterisk indicates statistically different values $(\mathrm{P}<0.05)$. $\mathbf{m}$ : Intact. $\square$ : Experimental cryptorchidism (Crypt.).

\section{Acknowledgments}

This study was supported in part by a Grant-in-Aid for Scientific Research from the Japan Society for the Promotion of Science 25292189 (to KM), and a grant from the INAMORI Foundation (to KM).

\section{References}

1. Murata S, Yashiroda H, Tanaka K. Molecular mechanisms of proteasome assembly. Nat Rev Mol Cell Biol 2009; 10: 104-115. [Medline] [CrossRef]

2. Tanaka K. The proteasome: overview of structure and functions. Proc Jpn Acad, Ser B, Phys Biol Sci 2009; 85: 12-36. [Medline] [CrossRef]

3. Saeki Y, Tanaka K. Assembly and function of the proteasome. Methods Mol Biol 2012; 832: 315-337. [Medline] [CrossRef]

4. Sahara K, Kogleck L, Yashiroda H, Murata $\mathbf{S}$. The mechanism for molecular assembly of the proteasome. Adv Biol Requl 2013; S2212-4926 00084-5. doi: 10. 1016/j. jbior.2013.09. 010. [Equb ahead of print]. 
A

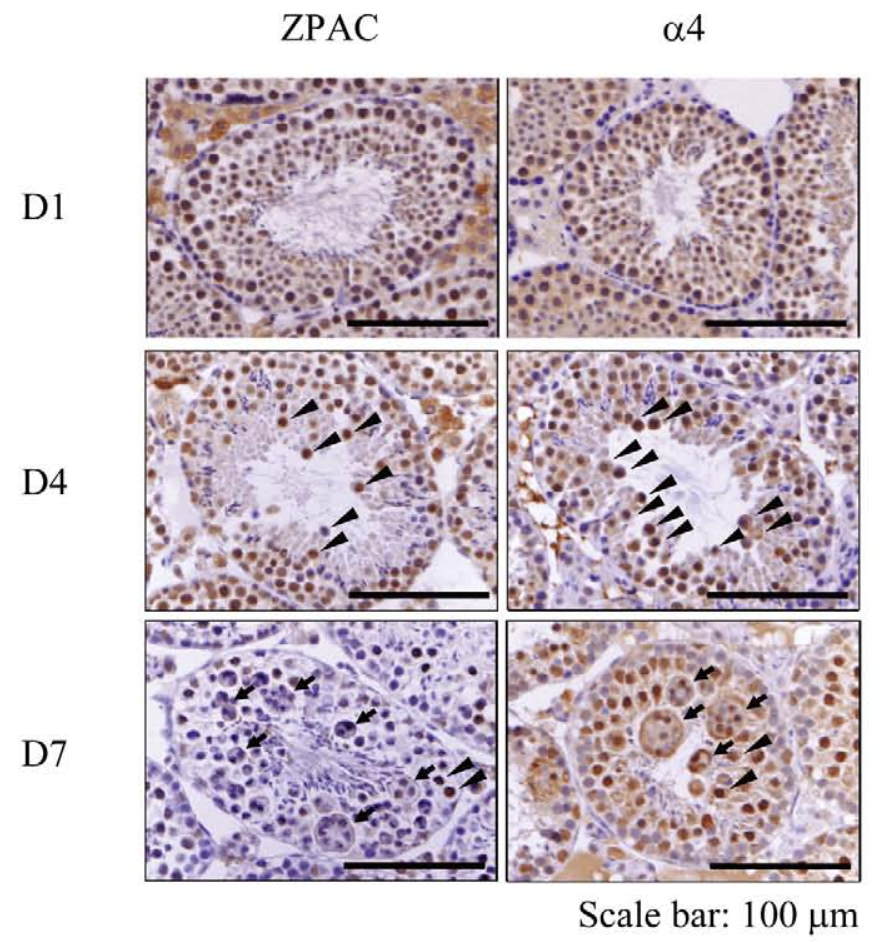

B


Scale bar: $100 \mu \mathrm{m}$

Fig. 4. Immunohistochemical analysis of ZPAC and $\alpha 4$ proteins in the cryptorchid mouse testis. (A) Cell-type- and developmental stage-specific localization of ZPAC and $\alpha 4$ proteins in the cryptorchid testis. ZPAC- or $\alpha 4$-positive cells in the seminiferous tubules are indicated by brown color. Each arrowhead indicates a representative ZPAC- or $\alpha 4$-positive cell with nuclear pyknosis/cellular shrinkage. Arrows indicate representative a4-positive multinucleated giant cells. Nonspecific staining of interstitial/Leydig cells is visible between seminiferous tubules (see in the Results section). Scale bars represent $100 \mu \mathrm{m}$. (B) Co-localization of ZPAC and annexin V in intact and cryptorchid testes at D1. Shown are representative images of testicular sections stained with DAPI (blue) and with anti-ZPAC and anti-annexin V antibodies (red and green, respectively). Arrowheads indicate representative cells double positive for ZPAC/annexin V. Nonspecific staining of interstitial/Leydig cells is visible between seminiferous tubules (see in the Results section). Scale bars represent $100 \mu \mathrm{m}$. 
5. Kunjappu MJ, Hochstrasser M. Assembly of the 20S proteasome. Biochim Biophys Acta 2014; 1843: 2-12. [Medline] [CrossRef]

6. Kniepert A, Groettrup M. The unique functions of tissue-specific proteasomes. Trends Biochem Sci 2014; 39: 17-24. [Medline] [CrossRef]

7. Shin SW, Shimizu N, Tokoro M, Nishikawa S, Hatanaka Y, Anzai M, Hamazaki J, Kishigami S, Saeki K, Hosoi Y, Iritani A, Murata S, Matsumoto K. Mouse zygotespecific proteasome assembly chaperone important for maternal-to-zygotic transition. Biol Open 2013; 2: 170-182. [Medline] [CrossRef]

8. Shin SW, Murata S, Matsumoto K. The ubiquitin-proteasome system in the maternal-tozygotic transition. J Mamm Ova Res 2013; 30: 79-85. [CrossRef]

9. Hou CC, Yang WX. New insights to the ubiquitin-proteasome pathway (UPP) mechanism during spermatogenesis. Mol Biol Rep 2013; 40: 3213-3230. [Medline] [CrossRef]

10. Crimmins S, Sutovsky M, Chen PC, Huffman A, Wheeler C, Swing DA, Roth K, Wilson J, Sutovsky P, Wilson S. Transgenic rescue of ataxia mice reveals a male-specific sterility defect. Dev Biol 2009; 325: 33-42. [Medline] [CrossRef]

11. Bedard N, Yang Y, Gregory M, Cyr DG, Suzuki J, Yu X, Chian RC, Hermo L, O'Flaherty C, Smith CE, Clarke HJ, Wing SS. Mice lacking the USP2 deubiquitinating enzyme have severe male subfertility associated with defects in fertilization and sperm motility. Biol Reprod 2011; 85: 594-604. [Medline] [CrossRef]

12. Ryu KY, Sinnar SA, Reinholdt LG, Vaccari S, Hall S, Garcia MA, Zaitseva TS, Bouley DM, Boekelheide K, Handel MA, Conti M, Kopito RR. The mouse polyubiquitin gene Ubb is essential for meiotic progression. Mol Cell Biol 2008; 28: 1136-1146. [Medline] [CrossRef]

13. Qian MX, Pang Y, Liu CH, Haratake K, Du BY, Ji DY, Wang GF, Zhu QQ, Song W, Yu Y, Zhang XX, Huang HT, Miao S, Chen LB, Zhang ZH, Liang YN, Liu S, Cha H, Yang D, Zhai Y, Komatsu T, Tsuruta F, Li H, Cao C, Li W, Li GH, Cheng Y, Chiba T, Wang L, Goldberg AL, Shen Y, Qiu XB. Acetylation-mediated proteasomal degradation of core histones during DNA repair and spermatogenesis. Cell 2013; 153: 1012-1024. [Medline] [CrossRef]

14. Yin Y, Hawkins KL, DeWolf WC, Morgentaler A. Heat stress causes testicular germ cell apoptosis in adult mice. $J$ Androl 1997; 18: 159-165. [Medline]

15. Bader M, Steller H. Regulation of cell death by the ubiquitin-proteasome system. Curr Opin Cell Biol 2009; 21: 878-884. [Medline] [CrossRef]

16. Rasoulpour RJ, Schoenfeld HA, Gray DA, Boekelheide K. Expression of a K48R mutant ubiquitin protects mouse testis from cryptorchid injury and aging. Am J Pathol 2003; 163: 2595-2603. [Medline] [CrossRef]

17. Kwon J, Wang YL, Setsuie R, Sekiguchi S, Sato Y, Sakurai M, Noda M, Aoki S, Yoshikawa Y, Wada K. Two closely related ubiquitin C-terminal hydrolase isozymes function as reciprocal modulators of germ cell apoptosis in cryptorchid testis. Am J Pathol 2004; 165: 1367-1374. [Medline] [CrossRef]

18. Kwon J, Mochida K, Wang YL, Sekiguchi S, Sankai T, Aoki S, Ogura A, Yoshikawa Y, Wada K. Ubiquitin C-terminal hydrolase L-1 is essential for the early apoptotic wave of germinal cells and for sperm quality control during spermatogenesis. Biol Reprod 2005; 73: 29-35. [Medline] [CrossRef]

19. Kazusa K, Namiki Y, Asano A, Kon Y, Endoh D, Agui T. Differences in spermatogen- esis in cryptorchid testes among various strains of mice. Comp Med 2004; 54: 179-184. [Medline]

20. Hirano Y, Hayashi H, Iemura S, Hendil KB, Niwa S, Kishimoto T, Kasahara M, Natsume T, Tanaka K, Murata S. Cooperation of multiple chaperones required for the assembly of mammalian 20S proteasomes. Mol Cell 2006; 24: 977-984. [Medline] [CrossRef]

21. Matsuoka T, Sato M, Tokoro M, Shin SW, Uenoyama A, Ito K, Hitomi S, Amano T, Anzai M, Kato H, Mitani T, Saeki K, Hosoi Y, Iritani A, Matsumoto K. Identification of ZAG1, a novel protein expressed in mouse preimplantation, and its putative roles in zygotic genome activation. J Reprod Dev 2008; 54: 192-197. [Medline] [CrossRef]

22. Satoh M, Tokoro M, Ikegami H, Nagai K, Sono Y, Shin SW, Nishikawa S, Saeki K, Hosoi Y, Iritani A, Fukuda A, Morimoto Y, Matsumoto K. Proteomic analysis of the mouse ovary in response to two gonadotropins, follicle-stimulating hormone and luteinizing hormone. J Reprod Dev 2009; 55: 316-326. [Medline] [CrossRef]

23. Tokoro M, Shin SW, Nishikawa S, Lee HH, Hatanaka Y, Amano T, Mitani T, Kato H, Anzai M, Kishigami S, Saeki K, Hosoi Y, Iritani A, Matsumoto K. Deposition of acetylated histones by RNAP II promoter clearance may occur at onset of zygotic gene activation in preimplantation mouse embryos. J Reprod Dev 2010; 56: 607-615. [Medline] [CrossRef]

24. Hatanaka Y, Shimizu N, Nishikawa S, Tokoro M, Shin SW, Nishihara T, Amano T, Anzai M, Kato H, Mitani T, Hosoi Y, Kishigami S, Matsumoto K. GSE is a maternal factor involved in active DNA demethylation in zygotes. PLOS ONE 2013; 8: e60205. [Medline] [CrossRef]

25. Russell LD, Ettlin RA, Sinha Hinkim AP, Clegg ED. Histological and histopathological evaluation of the testis. Florida: Cache River Press 1990; 41-58.

26. de Rooij DG. Stem cells in the testis. Int J Exp Pathol 1998; 79: 67-80. [Medline] [CrossRef]

27. Chaki SP, Misro MM, Ghosh D, Gautam DK, Srinivas M. Apoptosis and cell removal in the cryptorchid rat testis. Apoptosis 2005; 10: 395-405. [Medline] [CrossRef]

28. Print CG, Loveland KL. Germ cell suicide: new insights into apoptosis during spermatogenesis. BioEssays 2000; 22: 423-430. [Medline] [CrossRef]

29. Elliott MR, Zheng S, Park D, Woodson RI, Reardon MA, Juncadella IJ, Kinchen JM, Zhang J, Lysiak JJ, Ravichandran KS. Unexpected requirement for ELMO1 in clearance of apoptotic germ cells in vivo. Nature 2010; 467: 333-337. [Medline] [CrossRef]

30. Shaha C, Tripathi R, Mishra DP. Male germ cell apoptosis: regulation and biology. Philos Trans R Soc Lond B Biol Sci 2010; 365: 1501-1515. [Medline] [CrossRef]

31. Kubota H, Sasaki S, Kubota Y, Umemoto Y, Yanai Y, Tozawa K, Hayashi Y, Kohri K. Cyclooxygenase-2 protects germ cells against spermatogenesis disturbance in experimental cryptorchidism model mice. J Androl 2011; 32: 77-85. [Medline] [CrossRef]

32. Liu F, Huang H, Xu ZL, Qian XJ, Qiu WY. Germ cell removal after induction of cryptorchidism in adult rats. Tissue Cell 2012; 44: 281-287. [Medline] [CrossRef]

33. Li YC, Hu XQ, Xiao LJ, Hu ZY, Guo J, Zhang KY, Song XX, Liu YX. An oligonucleotide microarray study on gene expression profile in mouse testis of experimental cryptorchidism. Front Biosci 2006; 11: 2465-2482. [Medline] [CrossRef]

34. Shang $\mathbf{F}$, Taylor A. Ubiquitin-proteasome pathway and cellular responses to oxidative stress. Free Radic Biol Med 2011; 51: 5-16. [Medline] [CrossRef] 